\title{
Role of fibroblast growth factors in hepatocarcinogenesis of humans and rats Hermann and Bettina Grasl-Krauppbettina*
}

\author{
Address: Department of Medicine I, Institute of Cancer Research, Medical University of Vienna, Austria \\ Email: Bettina Grasl-Krauppbettina* - grasl-kraupp@meduniwien.ac.at \\ * Corresponding author
}

\author{
from I 3th Scientific Symposium of the Austrian Pharmacological Society (APHAR). Joint Meeting with the Austrian Society of Toxicology (ASTOX) and the \\ Hungarian Society for Experimental and Clinical Pharmacology (MFT) \\ Vienna, Austria. 22-24 November 2007 \\ Published: 14 November 2007 \\ BMC Pharmacology 2007, 7(Suppl 2):A57 doi:10.1 I86/I47|-2210-7-S2-A57
}

This abstract is available from: http://www.biomedcentral.com/I47/-22/0/7/S2/A57

(C) 2007 Gauglhofer et al; licensee BioMed Central Ltd.

The fibroblast growth factors (FGF) FGF-1 and FGF-2 are often deregulated in hepatocellular carcinoma (HCC). Information is missing on the role of other FGF-family members for hepatocarcinogenesis. We therefore determined the function of FGF-8, FGF-17 and FGF-18 in the development and progression of liver cancer. Half of the rat liver tumors studied showed enhanced expression of FGF-18. In about 50\% of human HCC, FGF-8, FGF-17 and FGF-18 and the corresponding FGF-receptors 3 and 4 were found to be upregulated. For functional studies we chose primary co-cultures of normal and premalignant (GSTp+) rat hepatocytes. DNA synthesis was significantly higher in GSTp + than in normal hepatocytes indicating an inherent growth advantage of the premalignant cell population. FGF-8 and FGF-18 stimulated DNA preferentially in GSTp+ hepatocytes, while FGF-17 exhibited no effect. In HCC-derived cell lines FGF-8, FGF-17 and FGF-18 stimulated growth, which involved phosphorylation of ERK1/ 2 and S6. Conclusions: FGF-8 and FGF-18 induced preferential growth of (pre)malignant hepatocytes and are highly upregulated in liver tumors indicating auto- and/or paracrine stimulation during formation and progression of the tumors. The results also show considerable similarities in the role of these FGFs in rat and human hepatocarcinogenesis implying that the molecular mechanisms underlying chemically induced rat hepatocarcinogenesis and human hepatocarcinogenesis are often identical. 